\title{
Fluoroquinolone resistance in multidrug- resistant Mycobacterium tuberculosis independent of fluoroquinolone use
}

\author{
To the Editor:
}

During the 1990s, multidrug-resistant tuberculosis (MDR-TB), resistant to at least isoniazid and rifampin, emerged as a great threat to global tuberculosis (TB) control [1]. For most MDR-TB patients, the World Health Organization (WHO) recommends a treatment regimen including second-line anti-TB drugs [2]. One of the most effective second-line drugs is fluoroquinolone [3]. During the treatment, MDR-TB may develop resistance to fluoroquinolone, or even become extensively drug-resistant (XDR-TB), which is resistant to both fluoroquinolone and at least one of three injectable second-line drugs [4]. The main genetic mechanism of fluoroquinolone resistance lies in the mutations in the quinolone-resistance-determining region of $\operatorname{gyr} A$ and $\operatorname{gyr} B$ [5].

A previous report has shown that less than $1 \%$ of pan-susceptible Mycobacterium tuberculosis isolates but often over 30\% of MDR M. tuberculosis isolates are fluoroquinolone resistant, a characteristic distribution leading to about $70 \%$ of fluoroquinolone resistance events and relevant marker gyr mutations in MDR M. tuberculosis isolates [6]. The development of fluoroquinolone resistance may be linked to its use. A meta-analysis study has demonstrated previous exposure to fluoroquinolone and thereafter fluoroquinolone resistance in M. tuberculosis [7]. Nevertheless, fluoroquinolone is widely used on farms, in veterinary clinics and in human healthcare facilities, making it difficult to obtain accurate information about prior use or exposure of patients [8].

During the study period, owing to a lack of $M$. tuberculosis culture for every TB patient, MDR-TB patients might experience various durations of drug-susceptible anti-TB regimens before MDR-TB is finally identified. Most new TB cases are given a drug-susceptible TB regimen and previously treated TB patients might be treated with first-line drugs plus second-line drugs, possibly including fluoroquinolone, before the drug susceptibility test has been completed. This situation has generated various randomly distributed durations for first-line drug regimens before MDR-TB is finally identified and a standard anti-MDR-TB regimen is started. Therefore, this study aims to explore to what degree the different regimens affect the development of fluoroquinolone resistance during treatment of MDR-TB.

During 2013-2015 we collected 2687 M. tuberculosis isolates from TB patients in Ningbo, a city in the mid-East of China with a population of 10 million. We tested susceptibility of these isolates in two steps using the BD BACTEC ${ }^{\text {TM }}$ MGITTM 960 system (Becton, Dickson and Company, NJ, USA) following WHO guidelines [9]. First, we tested susceptibility to the first-line anti-TB drugs isoniazid, rifampin, streptomycin and ethambutol. Based on the resistance results, we classified all isolates into three resistance subgroups: pan-susceptible (1593 isolates, susceptible to four first-line drugs), non-MDR (832 isolates, resistant to at least one first-line drug but not MDR) and MDR (262 isolates). Then, since most fluoroquinolone resistance events and relevant marker gyr mutations exist in the MDR group, the 262 MDR M. tuberculosis isolates were tested for susceptibility to ofloxacin, levofloxacin, moxifloxacin, kanamycin, capreomycin and amikacin, and amplified for the identification of gyr mutations as described previously [10]. Conversely, we estimated fluoroquinolone resistance and gyr mutations in 1593 pan-susceptible and 832 non-MDR resistant $M$. tuberculosis isolates by testing 100 randomly selected isolates in each group. In this way, we obtained the distribution of fluoroquinolone resistance in different groups with enough accuracy and an acceptable amount of labour.

@ERSpublications

Fluoroquinolone resistance in multidrug-resistant tuberculosis develops with various anti-tuberculosis regimens http://ow.ly/Rnfb30gv1xI

Cite this article as: Che Y, Song Q, Yang T, et al. Fluoroquinolone resistance in multidrug-resistant Mycobacterium tuberculosis independent of fluoroquinolone use. Eur Respir J 2017; 50: 1701633 [https:// doi.org/10.1183/13993003.01633-2017]. 
Finally, to assess the effect of the duration of different regimens on the development of fluoroquinolone resistance, we classified MDR $M$. tuberculosis isolates into groups according to treatment duration using intervals that allowed a similar number of isolates in each group. The prevalence of fluoroquinolone resistance among isolates from patients with less than 1 month of first-line drug treatment was used as the baseline prevalence, with prevalence in other groups being compared against this baseline (table 1).

We found 98 fluoroquinolone resistant isolates and $94 \mathrm{gyr}$ mutations in $262 \mathrm{MDR}$ M. tuberculosis isolates, three resistant isolates and three $g y r$ mutations in 100 non-MDR resistant isolates, and no fluoroquinolone resistance and gyr mutations in 100 randomly selected pan-susceptible isolates, respectively. Based on this prevalence, we estimated there were 25 fluoroquinolone-resistant isolates and $25 \mathrm{gyr}$ mutations in 832 non-MDR-resistant $M$. tuberculosis isolates. These results indicate that about $80 \%$ of fluoroquinolone-resistant isolates and gyr mutations exist in MDR M. tuberculosis isolates, a result similar to a previous report [6] and which justifies our strategy of studying fluoroquinolone resistance focusing on MDR M. tuberculosis isolates. Additionally, the 262 MDR-TB isolates were composed of 209 (fluoroquinolone resistant, 45\%) Beijing strains and 53 (fluoroquinolone resistant, 30\%) non-Beijing strains $(\mathrm{p}=0.20)$.

Our study shows that the prevalence of fluoroquinolone resistance in MDR M. tuberculosis isolates increases with the duration of the treatment regimen independent of whether the regimen contains first-line drugs or first-line drugs plus fluoroquinolone or injectable drugs (table 1). In patients with less than 1 month of first-line drug treatment, as much as $18 \%$ of MDR M. tuberculosis isolates were fluoroquinolone resistant. Compared with baseline, prevalence increased steadily with treatment duration. Except for a duration of $1-2$ months $(\mathrm{p}=0.14)$, the prevalence (range 38-62\%) was significantly higher $(p=0.03$ to $<0.001)$ than the baseline prevalence $(18 \%)$. Since no fluoroquinolone is usually used for new patients in the first few months of TB treatment, the increasing prevalence of fluoroquinolone resistance and gyr mutation may be associated with treatment duration, rather than use of fluoroquinolone. When patients treated for over 6 months were divided into two treatment groups, i.e. first-line drugs plus one fluoroquinolone and first-line drugs plus one injectable agent, the prevalence in the former group (62\%) was slightly greater than that in the latter group (52\%), showing no significant difference $(\mathrm{p}=0.63)$ and implying fluoroquinolone exposure because of treatment is not the only major cause of fluoroquinolone resistance and gyr mutation.

A previous study has demonstrated a higher than expected rate of MDR-TB in new patients never previously treated for TB, highlighting the extent to which MDR-TB has become an epidemic in its own way [11]. Since most fluoroquinolone-resistant strains come from MDR-TB patients, it is reasonable to speculate fluoroquinolone resistance also has its own way of reaching epidemic proportions from very early on. Also, a report has illustrated that of 53 patients with XDR-TB, 55\% claimed that they had never been treated, providing more evidence to indicate that there are factors other than fluoroquinolone

\begin{tabular}{|c|c|c|c|c|c|}
\hline $\begin{array}{l}\text { Duration } \\
\text { months }\end{array}$ & Regimen & $\begin{array}{c}\text { MDR } \\
\text { isolates }\end{array}$ & $\begin{array}{c}g y r \\
\text { mutations }\end{array}$ & $\begin{array}{l}\text { FQ-resistant } \\
\text { isolates }\end{array}$ & p-value ${ }^{\#}$ \\
\hline Total & & 262 & $94(36)$ & $98(37)^{\pi}$ & \\
\hline$<1$ & $H, R$ or $H, R, Z, E$ & 84 & $14(17)$ & $15(18)$ & Baseline \\
\hline $1-2$ & $H, R$ or $H, R, Z, E$ & 42 & $13(31)$ & $13(31)$ & 0.14 \\
\hline $3-6$ & $H, R$ or $H, R, Z, E$ & 50 & 19 (38) & 19 (38) & 0.03 \\
\hline 7-8 & $\begin{array}{l}\mathrm{H}, \mathrm{R} \text { or } \mathrm{H}, \mathrm{R}, \mathrm{Z}, \mathrm{E} \text {, plus one } \\
\text { injectable agent or } \mathrm{FQ}\end{array}$ & 54 & $28(52)$ & $29(54)$ & 0.002 \\
\hline $9+$ & $\begin{array}{l}H, R \text { or } H, R, Z, E \text {, plus one } \\
\text { injectable agent or } F Q\end{array}$ & 32 & 20 (62) & $22(69)$ & $<0.001$ \\
\hline $7+$ & $H, R$ or $H, R, Z, E$, plus one $F Q$ & 34 & $21(62)$ & $22(65)$ & $<0.001^{+, f}$ \\
\hline $7+$ & $\begin{array}{c}\mathrm{H}, \mathrm{R} \text { or } \mathrm{H}, \mathrm{R}, \mathrm{Z}, \mathrm{E} \text {, plus one } \\
\text { injectable agent }\end{array}$ & 52 & $27(52)$ & $29(56)$ & $<0.001^{+, f}$ \\
\hline
\end{tabular}

Data are presented as $\mathrm{n}$ or $\mathrm{n}(\%)$ unless otherwise stated. MDR: multidrug-resistant; XDR: extensively drug-resistant; $F Q$ : fluoroquinolone; $H$ : isoniazid; R: rifampicin; Z: pyrazinamide; E: ethambutol. \#: p-Value for $F Q$-resistance was calculated by comparing the prevalence of $F Q$-resistance for the treatment duration against the baseline prevalence; ๆ: Four cases that were phenotypically resistant but lacked detectable gyr mutations were $\mathrm{XDR}^{+}$: Data from regimens of 7-8 and 9+ month durations were combined to calculate statistics grouped by the use or absence of $\mathrm{FQ}{ }^{f}$ : The $\mathrm{p}$-value for comparison between the two 7+ month duration groups (with or without FQ treatment) was 0.63 . 
exposure in TB treatment that cause fluoroquinolone resistance [12]. Similar evidence was found in our study, i.e. four of $16 \mathrm{XDR}-\mathrm{TB}$ cases had no fluoroquinolone exposure. Very similar features also exist in the prevalence trend of the gyrA mutation. gyrA mutations are highly correlated with fluoroquinolone resistance (table 1), indicating that accumulating $g y r A$ mutations leads to increasing prevalence of fluoroquinolone resistance. D94G $(\mathrm{GAC} \rightarrow \mathrm{GGC})$ and $\mathrm{A} 90 \mathrm{~V}(\mathrm{GCG} \rightarrow \mathrm{GTG})$ gyrA mutations were most common, accounting for $80 \%$ of mutations. Among five types of gyr mutation, only the D94G $(\mathrm{GAC} \rightarrow \mathrm{GGC})$ gyrA mutation resulted in resistance to fourth-generation moxifloxacin [13], supporting previous reports that the $\mathrm{D} 94 \mathrm{G}$ mutation has a more significant effect on this compound [14].

However, our conclusion does not mean that fluoroquinolone use has no effect on resistance development. Instead, as shown by comparison between two subgroups of patients treated for over 6 months, fluoroquinolone use can slightly increase the prevalence of fluoroquinolone resistance. A similar weak relationship between fluoroquinolone resistance and fluoroquinolone consumption for $M$. tuberculosis has been demonstrated previously [15].

Our results show that MDR-TB patients experiencing a long period of irrational drug-susceptible regimens may increase the risk of becoming fluoroquinolone-resistant. Nowadays, in more and more areas of China, M. tuberculosis culture and drug susceptibility tests are stipulated for every TB patient. It will be less probable for MDR-TB patients to have a long period on a drug-susceptible regimen because of delay in identification of MDR-TB. However, there are still some factors that may cause delay, such as no rapid testing means in some hospitals, high expense and the time needed to get the first positive culture despite a positive smear test.

To summarise, this study shows that fluoroquinolone resistance develops with treatment of MDR-TB independent of whether regimens contain first-line drugs or first-line drugs plus fluoroquinolone or injectable drugs. This study also demonstrates that analysing fluoroquinolone resistance in MDR M. tuberculosis strains is an effective way to understand fluoroquinolone resistance in overall TB cases.

Yang Che, Qifa Song, Tianchi Yang, Guohua Ping and Mei Yu

Ningbo Municipal Centre for Disease Control and Prevention, Ningbo, Zhejiang, People's Republic of China.

Correspondence: Q. Song, Ningbo Municipal Centre for Disease Control and Prevention, 237 Yongfeng Road, Ningbo, Zhejiang 315010, People’s Republic of China. E-mail: songqf@nbcdc.org.cn

Received: July 262017 | Accepted after revision: Sept 142017

Support statement: This study was supported by the Natural Science Foundation of Ningbo (grant: 2015A610195). Funding information for this article has been deposited with the Crossref Funder Registry

Conflict of interest: None declared.

\section{References}

1 Falzon D, Schünemann HJ, Harausz E, et al. World Health Organization treatment guidelines for drug-resistant tuberculosis, 2016 update. Eur Respir J 2017; 49: 1602308.

2 Borisov SE, Dheda $\mathrm{K}$, Enwerem $\mathrm{M}$, et al. Effectiveness and safety of bedaquiline-containing regimens in the treatment of MDR- and XDR-TB: a multicentre study. Eur Respir J 2017; 49: 1700387.

3 Caminero JA, Sotgiu G, Zumla A, et al. Best drug treatment for multidrug-resistant and extensively drug-resistant tuberculosis. Lancet Infect Dis 2010; 10: 621-629.

4 Mitnick CD, Shin SS, Seung KJ, et al. Comprehensive treatment of extensively drug-resistant tuberculosis. $N$ Engl J Med 2008; 359: 563-574.

5 Pantel A, Petrella S, Veziris N, et al. Extending the definition of the GyrB quinolone resistance-determining region in Mycobacterium tuberculosis DNA gyrase for assessing fluoroquinolone resistance in M. tuberculosis. Antimicrob Agents Chemother 2012; 56: 1990-1996.

$6 \mathrm{Xu} \mathrm{P}, \mathrm{Li} \mathrm{X}$, Zhao M, et al. Prevalence of fluoroquinolone resistance among tuberculosis patients in Shanghai, China. Antimicrob Agents Chemother 2009; 53: 3170-3172.

7 van der Heijden YF, Maruri F, Blackman A, et al. Fluoroquinolone susceptibility in Mycobacterium tuberculosis after pre-diagnosis exposure to older- versus newer-generation fluoroquinolones. Int J Antimicrob Agents 2013; 42: 232-237.

8 Sukul P, Spiteller M. Fluoroquinolone antibiotics in the environment. Rev Environ Contam Toxicol 2007; 191: $131-162$.

9 Policy guidance on drug-susceptibility testing (DST) of second-line antituberculosis drugs: WHO/HTM/TB/ 2008.392. Geneva, World Health Organization, 2008. http://apps.who.int/iris/bitstream/10665/70500/1/WHO_ HTM_TB_2008.392_eng.pdf Date last accessed: November 13, 2017.

10 Devasia R, Blackman A, Eden S, et al. High proportion of fluoroquinolone-resistant Mycobacterium tuberculosis isolates with novel gyrase polymorphisms and a gyrA region associated with fluoroquinolone susceptibility. J Clin Microbiol 2012; 50: 1390-1396.

11 Nyang'wa BT, Brigden G, du Cros P, et al. Resistance to second-line drugs in multidrug-resistant tuberculosis. Lancet 2013; 381: 625 . 
12 Gandhi NR, Moll A, Sturm AW, et al. Extensively drug-resistant tuberculosis as a cause of death in patients co-infected with tuberculosis and HIV in a rural area of South Africa. Lancet 2006; 368: 1575-1580.

13 Gillespie SH. The role of moxifloxacin in tuberculosis therapy. Eur Respir Rev 2016; 25: 19-28.

14 Farhat MR, Jacobson KR, Franke MF, et al. Gyrase mutations are associated with variable levels of fluoroquinolone resistance in Mycobacterium tuberculosis. J Clin Microbiol 2016; 54: 727-733.

15 Shakoor S, Tahseen S, Jabeen K, et al. Fluoroquinolone consumption and -resistance trends in Mycobacterium tuberculosis and other respiratory pathogens: ecological antibiotic pressure and consequences in Pakistan, 2009-2015. Int J Mycobacteriol 2016; 5: 412-416. 